\title{
An Overview of Diagnostic Approach to Dengue Virus Infection
}

\author{
Muhammad Riaz ${ }^{1 *}$, Ghulam Rasool ${ }^{1}$, Junaid Akhtar ${ }^{1}$, Ghulam Muhammad Uttra ${ }^{1}$ and Sibghat Hasnain ${ }^{2}$ \\ ${ }^{1}$ Department of Allied Health Sciences, Sargodha Medical College, University of Sargodha, Pakistan \\ ${ }^{2}$ Department of Physiology, Sargodha Medical College, University of Sargodha, Pakistan
}

Submission:November 29, 2017; Published: April 12, 2018

*Corresponding author: Muhammad Riaz, Department of Allied Health Sciences, Sargodha Medical College, University of Sargodha, Pakistan, Email: riazmlt786@gmail.com

\begin{abstract}
Dengue fever is an infectious mosquito borne viral disease caused by dengue virus, an RNA virus from Flaviviridae family. The principal vector is the female mosquito Aedes aegypti. Infection with one dengue serotype provides lifelong immunity to patients against that serotype but only partial immunity to other dengue serotypes. Sign and symptoms vary in patients of classic dengue fever and dengue hemorrhagic fever. Various diagnostic approaches have been practiced since long time for the detection of dengue virus infection. Now a days, the most reliable approaches being practiced are the serological and molecular techniques. Enzyme Linked Immunosorbent Assay (ELISA) is most commonly used for the detection of dengue antibodies in serum of dengue patients while Polymerase Chain Reaction (PCR) is the most widely used molecular technique for the identification of dengue virus RNA and is considered as confirmatory approach for dengue viral infection. This review mainly discusses the diagnostic approaches for the detection of dengue virus in patients of classic dengue and dengue hemorrhagic fever.
\end{abstract}

Keywords: Dengue virus; Immunity; ELISA; PCR

\section{Introduction}

An infectious tropical disease called dengue fever is caused by the dengue virus [1]. Dengue virus (DENV) belongs to Flaviviridae family and is an RNA virus [2,3]. The four strains of dengue virus called dengue serotypes include DENV-1, DENV2, DENV-3 and DENV-4 causing full spectrum of the disease. An individual develop lifelong immunity against a particular serotype when infected for the first time but partial immunity against the other serotypes. In 2009, World Health Organization classified the dengue fever into uncomplicated and severe dengue fever [4] replacing the older classification established by WHO in 1997 in which dengue was classified into undifferentiated fever, dengue fever, and dengue hemorrhagic fever [5]. Further, dengue hemorrhagic fever was graded into grades I-IV. The presence of easy bruising with fever and positive tourniquet test dengue patients were included in grade-I while grade II dengue patients have spontaneous bleeding into skin or elsewhere and patients with clinical evidence of shock were placed in grade III whereas in grade IV dengue patients the shock is with severe hypotension and undetectable pulse rate [4].

An increase in dengue virus infection is reported in nearly 100 countries of the world including Asia, Americas, the Pacific, Africa, Thailand, Malaysia, Vietnam and Singapore. According to $\mathrm{CDC}$, nearly $40 \%$ of global population lives in dengue endemic areas. Each year, almost 100 million dengue cases with several hundred thousand dengue hemorrhagic cases require hospitalization [6]. The World Health Organization (WHO) estimated that among dengue virus infected cases particularly in children, 22000 deaths occur each year [4].

In Pakistan, dengue cases have been reported since the past two decades. In 1985, dengue has been documented for the first time [7] whereas the first major outbreak was reported in 1994-95. In 2006 following heavy rainfall, another dengue epidemic has been witnessed in Karachi [8]. In 2011 another major outbreak of dengue fever occurred in Lahore. According to Punjab Dengue Expert Committee reports, several thousand people were infected with dengue causing dozens of deaths. Among 29 deaths in Punjab province, 25 were from Lahore.

The transmission of dengue virus infection is primarily by Aedes mosquitoes, particularly A. aegypti while other disease transmitting Aedes species are A. albopictus, A. polynesiensis and A. scutellaris. Humans get infected when infective mosquito bites the healthy individual. After a person become infected with the mosquito, the virus undergoes an incubation period of 3 to 14 days (average, 4 to 7 days) leading to acute onset of fever following a variety of nonspecific signs and symptoms. During febrile phase (2-10 days of infection), the viruses may circulate 
in the peripheral blood. During this phase, infection may spread to uninfected persons if non infective A. aegypti mosquitoes bite the infected person become infective that subsequently transmits the virus to other uninfected persons, after an extrinsic incubation period of 8 to 12 days [9].

When an infective mosquito bites a person, the virus enters through the skin, binds to white blood cells and multiplies within cells throughout the body. In response the white blood cells produces a number of signaling proteins like interferon which are responsible for number of the symptoms, such as fever, severe pain and flu-like symptoms. In case of severe infection, increased virus multiplication inside the body affects many organs including liver and bone marrow causing leakage of fluid from blood stream through blood vessels into body cavities resulting severe hypotension. Furthermore, bone marrow dysfunction leads to reduction in platelets number which are vital to blood coagulation; thus increased risk of bleeding and other dengue fever complications [10].

\section{Diagnosis of Dengue Fever \\ Clinical findings}

Classic dengue fever mainly affects the adults and older children, characterized by the rapid onset of fever and a variety of symptoms, including retro-orbital pain, frontal headache, nausea and vomiting, body aches, joint pains, weakness and maculopapular rashes [11]. Anorexia, altered taste sensation, sore throat, diarrhea, constipation and respiratory symptoms are reported infrequently in classic dengue patients [12]. Dengue fever starts with headache, chills, pain upon moving the eyes, low backache, painful aching in the legs and joints with abrupt rise in temperature upto $104{ }^{\circ} \mathrm{F}\left(40{ }^{\circ} \mathrm{C}\right)$, bradycardia and hypotension [6]. Usually the rash appears on trunk spreading to the extremities and face with an average duration of 2 to 3 days. The end of the febrile phase leads to fall in temperature, appearance of petechiae, intense pruritus following desquamation on palms of the hands and soles of the feet. The most common symptoms are the petechiae and purpura with bleeding gums, menorrhagia, gastrointestinal (GI) hemorrhage and epistaxis [9].

Dengue hemorrhagic Fever (DHF) mainly affects the children under 15 years of age and is characterized by rapid onset of fever and headache usually lasts for 2 to 7 days [13] causing hemorrhage (bleeding), abdominal pain, and circulatory collapse (shock). Respiratory and intestinal symptoms may appear along with sore throat, nausea, vomiting, cough, and abdominal pain. After DHF symptoms appear, shock occurs 2 to 6 days with sudden collapse, weak pulse, clammy extremities and circumoral cyanosis (blueness around the mouth). In DHF, there is bleeding with easy bruising, petechiae (blood spots in the skin), hematemesis (spitting up blood), melena (blood in the stool), gum bleeding, and epistaxis. Vascular leakage in DHF results in serous effusions and hemoconcentration leading to circulatory collapse [14].
It is very difficult to differentiate between DHF from classical dengue fever during acute phase of illness, in which the differential diagnosis should include measles, influenza, rubella, leptospirosis, typhoid, malaria and other viral fevers. During acute stage no pathognomic symptoms and signs of DHF appears while in the critical stage sign of circulatory failure may occur within 24 hours before or after the temperature falls to normal or below (Figure 1). The plasma leakage in dengue fever patients leads to dengue shock syndrome resulting in severe hypotension and undetectable pulse rate [15]. The patients in dengue shock syndrome may die within 8 to $24 \mathrm{~h}$ while antishock therapy may lead to rapid recovery usually 2 to 3 days [9].

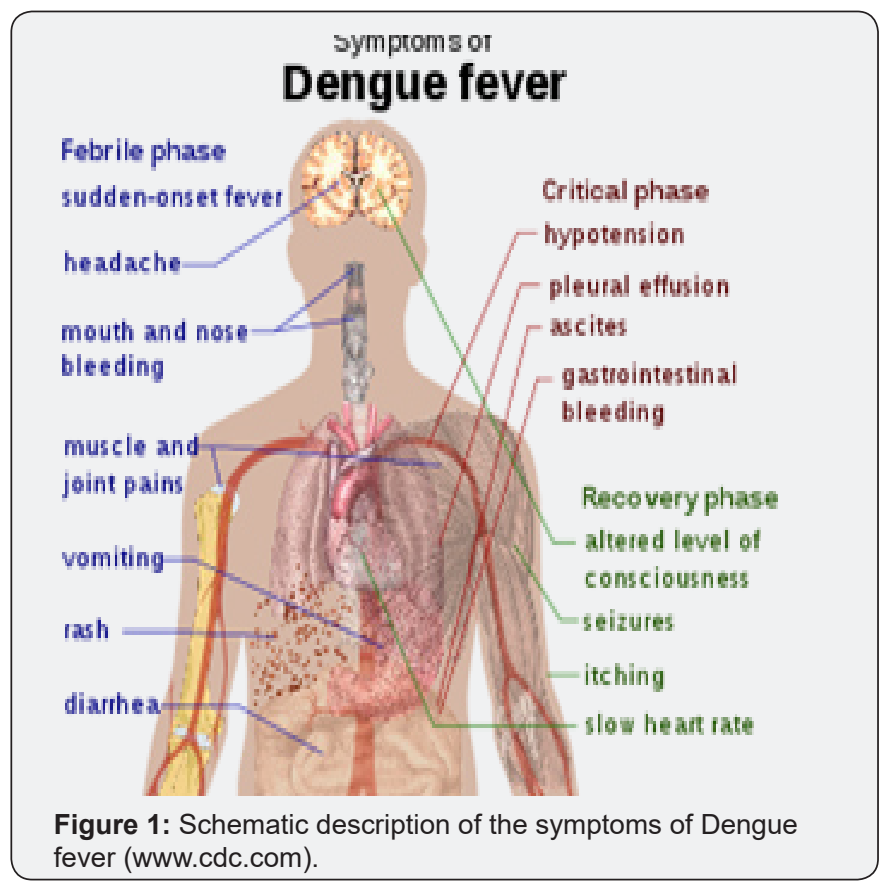

\section{Laboratory Findings}

\section{Torniquet test}

The tourniquet test is performed at Physicians clinic in which the pressure is applied by inflating the blood pressure cuff between diastolic and systolic blood pressure on the upper arm for 5 minutes [16]. Appearance of 20 petechiae per square inch on the skin area under pressure indicates positive tourniquet test [5].

\section{Hematological findings}

In dengue fever, complete blood picture demonstrates lower total WBC Count and neutrophils. During febrile phase of illness, reduction in lymphocytes is common but increased lymphocyte count with atypical cells is oftenly observed after febrile phase of dengue virus infection and before shock [17]. Increased hematocrit more than $20 \%$ is a sign of hemoconcentration. Regular monitoring of hematocrit should be performed at least every $24 \mathrm{~h}$ in dengue fever and every 3-4 $\mathrm{h}$ in dengue shock syndrome. Platelet count is also reduced in dengue virus infection $(<100,000$ cells $/ \mu \mathrm{L}$ in dengue shock syndrome) and should be 
monitored every 24h [4]. Prothrombin time (PT) and activated partial thromboplastin time (APTT) are usually prolonged while fibrinogen level is reduced in dengue virus infection [6].

\section{Biochemical findings}

The most common electrolyte abnormality is the reduction of sodium level in patients of DHF or DSS (dengue shock syndrome) [3]. Elevated levels of blood urea nitrogen (BUN) are observed in patients with DHF. In patients of dengue hemorrhagic fever with acute hepatitis, there observed elevation of liver aminotransferases from mild to several thousands. Hypoalbuminemia is a sign of hemoconcentration in dengue patients [18].

\section{Serological findings}

Serologic tests routinely used in diagnosing dengue infection are hemagglutination-inhibition (HI) test, neutralization test (NT), complement fixation (CF), immunoglobulin M (IgM) capture enzyme-linked immunosorbent assay (MAC-ELISA), and indirect immunoglobulin G ELISA [19]. Hemagglutination Inhibition (HI) test has been used most frequently for dengue diagnosis and is very sensitive, reliable and easily performed with minimal instrumental requirements. HI antibodies persist for a longer period usually upto 48 years which begins to appear on 5 th or 6 th days of illness. The only major disadvantage of HI test is its non specificity making this test unreliable in identifying the viral serotype [16].

The compliment fixation (CF) test is less commonly used serologic test for dengue diagnosis due to the requirement of highly trained personnel. The CF test is based on the principle that during antigen-antibody reactions the compliment is consumed. This test is more specific in primary infection and persists for shorter periods [16].

The neutralization test (NT) is another serologic test used for the diagnosis of dengue virus infection which is most commonly used test in dengue laboratories. NT is more sensitive and specific to neutralizing antibodies which are present even in the absence of detectable $\mathrm{HI}$ antibodies in patients with past dengue infection. The disadvantages are time consuming, expensive and require technically skilled personnel [19].

During the past few years, most widely used serologic test for dengue diagnosis is the Enzyme Linked Immunosorbent Assay (ELISA) particularly for the detection of IgM. The IgM capture enzyme-linked immunosorbent assay (MAC-ELISA) is a simple rapid test that requires sophisticated equipment [20]. In acute phase of dengue infection anti-dengue IgM antibody develops followed by IgG antibody and in most of the patients IgM antibody become detectable by day 5 of illness. With both primary and secondary dengue infection, IgM antibody is produced and this may persists for more than 90 days in some of primary infections while in most patients the level of IgM become undetectable by 60 days of infection [21]. An indirect IgG-ELISA has been developed to differentiate primary dengue infection from secondary dengue infections which is very simple and easily performed. The IgG-ELISA cannot be used for the identification of infecting dengue virus serotype due to its nonspecificity and broad cross-reactivity among flaviviruses [21].

\section{Molecular diagnosis}

For the serotype specific diagnosis of dengue fever, Reverse transcriptase PCR (RT-PCR) has revolutionized the laboratory diagnosis. RT-PCR is a simple, rapid, sensitive and reproducible test used for the detection of viral RNA [4]. RT-PCR has become a primary tool for the detection of viral RNA in acute phase of illness with sensitivity ranging from $80-90 \%$ and specificity of more that $95 \%$. A positive PCR result usually confirms the infecting serotype and provides a definite proof of current infection. However, a negative PCR result before 5 days of illness is interpreted as "indeterminate" and such patients are usually asked for serological confirmation by submitting second serum sample after 5th day of illness [6].

\section{Imaging studies}

Ultrasonography is a cost effective imaging technique used for the diagnostic evaluation of dengue hemorrhagic fever [22]. The findings of positive ultrasonography of dengue patients include fluid in abdominal and chest cavities, thickened gall bladder wall and pericardial effusion. Plasma leakage has been observed in some dengue patients within three days of the onset of fever [23]. Chest radiography is another imaging technique used to look for bronchopneumonia and pleural effusions. In patients of dengue shock syndrome, bilateral pleural effusions are common with typical right-sided pleural effusion. For the detection of intracranial bleeding or cerebral edema in patients of dengue hemorrhagic fever, head computed tomography can be used without contrast is indicated in dengue patients with distorted consciousness [6].

\section{Histological findings}

Biopsy of the skin lesions in patients with classic dengue infection reveals small blood vessels anomaly. The primary histologic findings from skin lesions of dengue patients are perivascular edema, endothelial swelling, and mononuclear cell infiltration [6].

\section{Treatment}

No specific antiviral medication is required for patients suffering from dengue fever. To prevent dehydration due to fever, vomiting or lack of fluid intake; the patients are encouraged to drink rehydration fluids, water and fruit juices. The dengue patients are encouraged to take acetaminophen (paracetamol) to get relief from pain and fever. Patients of classic dengue fever and dengue hemorrhagic fever should avoid the use of nonsteroidal anti-inflammatory drugs (NSAIDs). Intravenous volume replacement may be given to patients with dengue shock syndrome or dengue hemorrhagic fever [6]. 


\section{References}

1. Whitehorn J, Farrar J (2010) Dengue. Br Med Bull 95: 161-173.

2. Gould EA, Solomon T (2008) Pathogenic flaviviruses. The Lancet 371(9611): 500-509.

3. Syed S, Mahmood Z, Riaz S, Latif N, Majeed A Rashid (2014) Elemental profile of blood serum of dengue fever patients from Faisalabad, Pakistan. IJCBS 6(2014): 34-37.

4. World Health Organization (2009) Dengue Guidelines for Diagnosis, Treatment, Prevention and Control. Geneva, Switzerland.

5. World Health Organization (1997) Dengue haemorrhagic fever: diagnosis, treatment, prevention and control. ( $2^{\text {nd }}$ Edn). Geneva, Switzerland.

6. Center for Disease Control and Prevention (CDC) (2010) Dengue Fever (DF) and Dengue Hemorrhagic Fever (DHF)". Yellow Book. pp. 12-23.

7. Ansari J K, Siddiq M, Hussain T, Baig I, Tariq WZ (2001) Outbreak of Dengue Haemorrhagic Fever in Karachi. Pak Armed Forces Med J 51: $94-98$.

8. Ahmed S, Arif F, Yahya Y, Rehman A, Abbas K, et al. (2008) Dengue fever outbreak in Karachi 2006 - A study of profile and outcome of children under 15 years of age. J Pak Med Assoc 58(1): 4-8.

9. Duane JG (1998) Dengue and Dengue Hemorrhagic Fever. Clin Microbiol Reviews 11(3): 480-496.

10. Martina BEE, Koraka P, Osterhaus ADME (2009) Dengue Virus Pathogenesis: an Integrated View. Clin Microbiol Rev 22(4): 564-581.

11. Hayes EB, Gubler DJ (1992) Dengue and dengue hemorrhagic fever. Pediatr Infect Dis J 11(4): 311-317.

12. Statler J, Mammen M, Lyons A, Sun W (2008) Sonographic findings of healthy volunteers infected with dengue virus. J Clin Ultrasound 36(7): 413-417.

This work is licensed under Creative

Commons Attribution 4.0 License

DOI: 10.19080/CTBEB.2018.13.555872
13. Dietz V, Gubler DJ, Ortiz S, Kuno G, Casta Velez A, et al. (1996) The 1986 dengue and dengue hemorrhagic fever epidemic in Puerto Rico: epidemiologic and clinical observations. P R Health Sci J 15(3): 201210.

14. Halstead SB (2007) Dengue. Lancet 370(9599): 1644-1652.

15. Anonymous (1986) Dengue hemorrhagic fever, diagnosis, treatment and control. World Health Organization, Geneva, Switzerland.

16. Gubler DJ, Sather GE (1988) In: Homma A, Cunha JF (Eds.), Proceedings of the International Symposium on Yellow Fever and Dengue. Laboratory diagnosis of dengue and dengue hemorrhagic fever. pp. 291-322.

17. Potts JA, Rothman AL (2008) Clinical and laboratory features that distinguish dengue from other febrile illnesses in endemic populations. Trop Med Int Health 13(11): 1328-1340.

18. Lima EQ Nogueira ML (2008) Viral hemorrhagic fever induced acute kidney injury. Semin Nephrol 28(4): 409-415.

19. Guzma MG, Kouri G (1996) Advances in dengue diagnosis. Clin Diagn Lab Immunol 3(6): 621-627.

20. Centers for Disease Control and Prevention (2010) Dengue Laboratory Guidance and Diagnostic Testing.

21. Domingo C, de Ory F, Sanz JC, Reyes N, Gascon J, et al. (2009) Molecular and serologic markers of acute dengue infection in naive and flavivirus vaccinated travelers. Diagn Microbiol Infect Dis 65(1): 42-48.

22. Qureshi JA, Notta NJ, Salahuddin N, Zaman V, Khan JA (1997) An epidemic of Dengue fever in Karachi. associated clinical manifestations. J Pak Med Assoc 47(7): 178-181.

23. Srikiatkhachorn A, Krautrachue A, Ratanaprakarn W, Wongtapradit L, Nithipanya N, et al. (2007) Natural history of plasma leakage in dengue hemorrhagic fever: a serial ultrasonographic study. Pediatr Infect Dis J 26(4): 283-290.

Your next submission with Juniper Publishers will reach you the below assets

- Quality Editorial service

- Swift Peer Review

- Reprints availability

- E-prints Service

- Manuscript Podcast for convenient understanding

- Global attainment for your research

- Manuscript accessibility in different formats ( Pdf, E-pub, Full Text, Audio)

- Unceasing customer service

Track the below URL for one-step submission

https://juniperpublishers.com/online-submission.php 